Algebraic 83 Geometric $\mathcal{T}_{\text {opology }}$

Volume 5 (2005) 23-29

Published: 7 January 2005

Corrected: 22 June 2005

ATG

\title{
Poincaré submersions
}

\author{
JOHN R. KLEIN
}

\begin{abstract}
We prove two kinds of fibering theorems for maps $X \rightarrow P$, where $X$ and $P$ are Poincaré spaces. The special case of $P=S^{1}$ yields a Poincaré duality analogue of the fibering theorem of Browder and Levine.
\end{abstract}

AMS Classification 57P10; 55R99

Keywords Poincaré duality space, fibration

\section{Introduction}

One of the early successes of surgery theory was the fibering theorem of Browder and Levine [B-L, which gives criteria for when a smooth map $f: M \rightarrow S^{1}$ is homotopic to a submersion. Here $M$ is assumed to be a connected closed, smooth manifold of dimension $\geq 6$, and we also require $f$ to induce an isomorphism of fundamental groups. The Browder-Levine fibering theorem then says that $f$ is homotopic to a submersion if and only if the homotopy groups of $M$ are finitely generated in each degree.

The purpose of the current note is to prove fibering results in the Poincaré duality category. Note that a submersion of closed manifolds is a smooth fiber bundle with closed manifold fibers. Replacing the closed manifolds with finitely dominated Poincaré spaces and the fiber bundle with a fibration yields the notion of Poincaré submersion: this is a map between Poincaré spaces whose homotopy fibers are Poincaré spaces.

Our first result concerns the case when the target is acyclic (this includes the Browder-Levine situation). Let $X$ be a connected, finitely dominated Poincaré duality space of (formal) dimension $d$ and fundamental group $\pi$. Let

$$
f: X \rightarrow B \pi
$$

be the classifying map for the universal cover of $X$. We will be assuming that the classifying space $B \pi$ is a finitely dominated Poincaré space of dimension $p$. 
Theorem A Let $F$ denote the homotopy fiber of $f$. Then $F$ is a homotopy finite Poincaré duality space of dimension $d-p$ if and only if the homotopy groups of $X$ are finitely generated in each degree.

For our second result, let $f: X \rightarrow P$ be a map of orientable, finitely dominated and connected Poincaré duality spaces. Assume $X$ has dimension $d$ and $P$ has dimension $p$. We will give criteria for deciding when the homotopy fiber $F$ of $f$ satisfies Poincaré duality.

Let $i: F \rightarrow X$ be the evident map. There is an umkehr homomorphism

$$
i_{*}^{!}: H_{*}(X) \rightarrow H_{*-p}(F)
$$

which is defined if $p \geq 3$ or if $P$ is 1 -connected (cf. $\S 4$ ). The pushforward of a fundamental class $[X] \in H_{d}(X)$ for $X$ with respect to $i^{!}$then gives a class

$$
x_{f}:=i_{*}^{!}([X]) \in H_{d-p}(F) .
$$

This will be our candidate for a fundamental class of $F$.

Theorem B Assume that $f$ is 2-connected. Then the following are equivalent:

(1) $H_{*}(F)=0$ in sufficiently large degrees 1

(2) $F$ is homotopy finite.

(3) $F$ is a Poincaré duality space.

If in addition $X$ is 1-connected, then the above are equivalent to the assertion that

(4) the homomorphism

$$
\cap x_{f}: H^{*}(F) \rightarrow H_{d-p-*}(F)
$$

is an isomorphism in all degrees.

Remark When $P=S^{p}$ is a sphere, $(1) \Rightarrow(3)$ overlaps with [C, lemma 1.1]. The implication $(2) \Rightarrow(3)$ is a consequence of [K11, theorem B].

We do not a priori assume that Poincaré duality spaces satisfy a finiteness condition, so the implication $(3) \Rightarrow(2)$ is non-trivial.

\footnotetext{
${ }^{1}$ Correction added June, 2005: If $X$ is not 1 -connected, one also requires the hypothesis that the homotopy groups of $X$ are finitely generated. I am indebted to Jonathan Hillman for pointing out that a hypothesis was missing here. Hillman also communicated to me the following counterexample: take $X$ to be the connected sum of $S^{5} \times S^{1}$ with $S^{3} \times S^{3}$ and let $f: X \rightarrow S^{1}$ classify the universal cover. Then $\pi_{3}(F)$ is infinitely generated.
} 
Conventions A space is homotopy finite if has the homotopy type of a finite cell complex. A space is finitely dominated if it is the retract of a homotopy finite space.

A Poincaré space of formal dimension $d$ is a space $X$ for which there exists a pair $(\mathcal{L},[X])$ consisting of a rank one abelian system of local coefficients $\mathcal{L}$ on $X$ and a (fundamental) class $[X] \in H_{d}(X ; \mathcal{L})$ such that the cap product homomorphism

$$
\cap[X]: H^{*}(X ; \mathcal{A}) \rightarrow H_{d-*}(X ; \mathcal{L} \otimes \mathcal{A})
$$

is an isomorphism, for all local coefficient modules $\mathcal{A}$ on $X$ (cf. W1, Kl2 ). If $X$ is connected, then it is enough to establish the isomorphism when $\mathcal{A}$ is the integral group ring of the fundamental group of $X$. When the local system $\mathcal{L}$ is constant, we say that $X$ is orientable. We do not at assume any finiteness conditions in the definition of Poincaré space appearing here. However, in the 1-connected case, homotopy finiteness is actually a consequence of Poincaré duality (see 3.2 below).

Acknowledgements The author is indebted to Mokhtar Aouina, Graeme Segal and Andrew Ranicki for the discussions that led to this paper.

The author was partially supported by NSF Grant DMS-0201695.

\section{Proof of Theorem $\AA$}

We first prove the 'only if' part. Assume that $F$ is a homotopy finite Poincaré space. Since $F$ is 1-connected and homotopy finite, we infer that its homology is finitely generated. Apply the $\bmod \mathcal{C}$ Hurewicz theorem (with $\mathcal{C}=$ the Serre class of finitely generated abelian groups) to see that the homotopy groups of $F$ are finitely generated [S, corollary 9.6.16].

We now prove the 'if' part. Note that $F$ has the homotopy type of the universal cover of $X$, so $F$ is homotopy finite dimensional bacause $X$ is. By the long exact homotopy sequence and the fact that $\pi_{*}(X)$ is degreewise finitely generated, we infer that $\pi_{*}(F)$ is degreewise finitely generated. Since $F$ is simply connected, the mod $\mathcal{C}$ Hurewicz theorem shows that the homology groups of $F$ are finitely generated. By a result of Wall W2, we see that $F$ is homotopy finite.

We now know that each space in the homotopy fiber sequence

$$
F \rightarrow X \rightarrow B \pi
$$


is finitely dominated. It follows directly from [K11, theorem B] (see also [G] that $F$ satisfies Poincaré duality and has formal dimension $d-p$. This completes the proof of Theorem A.

\section{Duality and finiteness}

A chain complex $C$ of abelian groups is said to be dualizable if there is chain complex $D$ and a map

$$
d: \mathbb{Z} \rightarrow C \otimes D
$$

$(\otimes=$ derived tensor product, and $d$ is allowed to be degree shifting) such that, for all $P$, we get that the induced map of complexes

$$
\operatorname{hom}(C, P) \rightarrow \operatorname{hom}(\mathbb{Z}, P \otimes D)
$$

(derived hom) given by $f \mapsto\left(f \otimes 1_{D}\right) \circ d$ induces an isomorphism on homology, where $1_{D}$ denote the identity map of $D$.

A chain map $C \rightarrow D$ is said to be a weak equivalence if it induces an isomorphism in homology. More generally $C$ and $D$ are said to be weak equivalent if there is a finite sequence of weak equivalences starting at $C$ and ending at $D$. A chain complex is (chain) homotopy finite if it is weak equivalent to a finite chain complex, i.e., a complex of finite rank free abelian groups with finitely many non-trivial degrees. A chain complex is finitely dominated if is a retract up to homotopy of a finite chain complex. It is well-known chain complex over $\mathbb{Z}$ is homotopy finite if and only if it is finitely dominated (see [W2]).

Lemma 3.1 If $C$ is dualizable, then it is homotopy finite over $\mathbb{Z}$.

Proof Since $\mathbb{Z}$ is "compact," there exists a finite chain complex $C_{0}$, a map $i: C_{0} \rightarrow C$ and a map $d_{0}: \mathbb{Z} \rightarrow C_{0} \otimes D$ such that

$$
\mathbb{Z} \stackrel{d_{0}}{\longrightarrow} C_{0} \otimes D \stackrel{i \otimes 1}{\longrightarrow} C \otimes D
$$

is homotopic to $d$. Consider the homotopy commutative diagram

$$
\begin{array}{ccc}
\operatorname{hom}(C, C) & \stackrel{\left(-\otimes 1_{C}\right) \circ d}{\simeq} & \operatorname{hom}(\mathbb{Z}, C \otimes D) \\
i_{*} \uparrow & \uparrow_{i_{*}} \\
\operatorname{hom}\left(C, C_{0}\right) \underset{\left(-\otimes 1_{C}\right) \circ d}{\stackrel{\simeq}{\longrightarrow}} & \operatorname{hom}\left(\mathbb{Z}, C_{0} \otimes D\right)
\end{array}
$$

Algebraic 8 G Geometric Topology, Volume 5 (2005) 
The map $d_{0}$ lives in the lower right corner and maps to $d$ under the right vertical map. The map $1_{C}$ maps to $d$ under the top horizontal map. Since the lower horizontal map is an equivalence, we get a map $j: C \rightarrow C_{0}$ such that $i_{*}(j)=j \circ i$ is homotopic to $1_{C}$. We conclude that the identity map of $C$ factors up to homotopy through the finite object $C_{0}$.

Note now if $X^{d}$ is a 1-connected space which is equipped with a chain level fundamental class $[X]$ for which Poincaré duality holds, then $C(X)=$ the singular chains on $X$ is dualizable using the maps

$$
\mathbb{Z} \stackrel{[X]}{\longrightarrow} C(X) \stackrel{\text { diagonal }}{\longrightarrow} C(X \times X) \simeq C(X) \otimes C(X),
$$

where the first map is the homomorphism (of degree $d$ ) induced by a choice of fundamental class. By the above lemma, we infer that $C(X)$ is homotopy finite.

A result of Wall says that a 1-connected space is homotopy finite if and only if its chain complex is (chain) homotopy finite (see [W3]). Hence,

Corollary 3.2 Let $X$ be a 1-connected space which satisfies Poincaré duality. Then $X$ is also homotopy finite.

\section{The umkehr homomorphism}

According to W1, theorem 2.4], if $\operatorname{dim} P \geq 3$ is a Poincaré duality space, then there is a homotopy equivalence

$$
P \simeq P_{0} \cup_{\alpha} D^{p}
$$

in which $P_{0}$ is a $\mathrm{CW}$ complex of dimension $\leq p-1$. If $P$ is 1 -connected, then $P_{0}$ has the homotopy type of a CW complex of dimension $\leq p-2$. If $P$ has dimension $\leq 2$, then $P \simeq S^{p}$, and the above decomposition is also available.

Furthermore, once an orientation for $P$ has been chosen, the above cell decomposition is unique up to oriented homotopy equivalence. ¿From now on, we fix an identification $P:=P_{0} \cup D^{p}$, where $\operatorname{dim} P_{0} \leq p-1$.

Without loss in generality, let us assume that $f: X \rightarrow P$ has been converted into a Hurewicz fibration. Let $X_{0}=f^{-1}\left(P_{0}\right)$. Then we obtain a pushout square

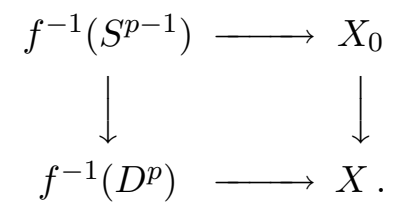

Algebraic 8 G Geometric Topology, Volume 5 (2005) 
Using the homotopy lifting property, we see that the pair $\left(f^{-1}\left(D^{p}\right), f^{-1}\left(S^{p-1}\right)\right)$ has the homotopy type of the pair $\left(F \times D^{p}, F \times S^{p-1}\right)$. Taking vertical cofibers in the diagram, we get an umkehr map

$$
i^{!}: X \longrightarrow X / X_{0}=f^{-1}\left(D^{p}\right) / f^{-1}\left(S^{p-1}\right) \simeq F_{+} \wedge S^{p}
$$

The umkehr homomorphism

$$
i_{*}^{!}: H_{*}(X) \rightarrow H_{*-p}(F)
$$

is the effect of applying singular homology to $i^{!}$, and using the suspension isomorphism to perform the degree shift.

\section{Proof of Theorem $B$}

$(1) \Rightarrow(2) \quad$ By the long exact homotopy sequence of the fibration, we see that $\pi_{*}(F)$ is degreewise finitely generated. By the $\bmod \mathcal{C}$ Hurewicz theorem, we infer that $H_{*}(F)$ is finitely generated. Then $F$ is homotopy finite by W2.

$(2) \Rightarrow(3)$ Follows from [Kl1, theorem B].

$(3) \Rightarrow(1) \quad$ This follows from 3.2

For the remainder of the proof of the theorem, we suppose that $X$ is 1connected. Then so are $F$ and $P$.

$(3) \Rightarrow(4)$ It will be enough to show that the class $x_{f}$ is a generator of $H_{d-p}(F) \cong \mathbb{Z}$. By definition of $x_{f}$, this is equivalent to knowing that the homomorphism

$$
i_{*}^{!}: H_{d}(X) \rightarrow H_{d-p}(F)
$$

is of degree \pm 1 .

This can be seen as follows: the space $X_{0}$ is the pullback of the fibration $f: X \rightarrow P$ along a CW complex $P_{0}$ of dimension $\leq p-2$ (this uses the fact that $P$ is 1 -connected, cf. $\S 4$ ). As $F$ has formal dimension $\leq d-p$, it is straightforward to check that $X_{0}$ has the homotopy type of a CW complex of dimension $\leq d-2$. Using the homotopy cofiber sequence

$$
X_{0} \longrightarrow X \stackrel{i^{!}}{\longrightarrow} F_{+} \wedge S^{p}
$$

and the fact that the homology of $X_{0}$ vanishes above degree $d-2$, we see that $i^{!}$induces an isomorphism in homology in degree $d$.

$(4) \Rightarrow(3) \quad$ Trivial. 


\section{References}

[B-L] Browder W., Levine J.: Fibering manifolds over a circle. Comment. Math. Helv. 40 153-160 (1966). MathReview

[C] Casson A. J.: Fibrations over spheres. Topology 6 489-499 (1967). MathReview

[G] Gottlieb, D. H.: Poincaré duality and fibrations. Proc. Amer. Math. Soc. 76, 148-150 (1979). MathReview

[Kl1] Klein, J. R.: The dualizing spectrum of a topological group. Math. Ann. 319, 421-456 (2001). MathReview

[K12] Klein, J. R.: Poincaré duality spaces. Surveys on surgery theory, Vol. 1, 135-165 Ann. of Math. Stud. 145, Princeton Univ. Press 2000 MathReview

[S] Spanier, E. H.: Algebraic Topology. McGraw-Hill 1966 MathReview

[W1] Wall, C. T. C.: Poincaré complexes: I. Ann. of Math. 86, 213-245 (1970) MathReview

[W2] Wall, C. T. C.: Finiteness conditions for CW-complexes. Ann. of Math. 81 56-69 (1965). MathReview

[W3] Wall, C. T. C.: Finiteness conditions for CW complexes. II. Proc. Roy. Soc. Ser. A 295 129-139 (1966). MathReview

Department of Mathematics, Wayne State University

Detroit, MI 48202, USA

Email: klein@math.wayne.edu

Received: 15 November 2004 